\title{
UDC 621.039.619 \\ DESIGN AND RESEARCH PROGRAM OF THE ALIANCE-T EXPERIMENT
}

(short message)

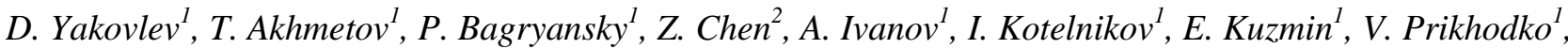 \\ I. Shikhovtsev ${ }^{l}, Q$. Zeng $^{2}$
}

\footnotetext{
${ }^{1}$ Budker Institute of Nuclear Physics of Siberian Branch Russian Academy of Sciences, Novosibirsk, 630090, Russia

${ }^{2}$ Institute of Nuclear Energy Safety Technology, Hefei Institutes of Physical Science, Chinese Academy of Sciences, Hefei, Anhui, 230031, China
}

ALIANCE-T is a pilot experiment aiming to test the core principles of operation of the ALIANCE [1] volumetric fusion neutron source and to study specific plasma physics problems related to continuously operating gas dynamic mirror traps [2]. Research program of ALIANCE-T focuses on achievement of continuous discharge in axisymmetric magnetic field with high mirror ratio $(\sim 100)$ with parameters of plasma suitable for neutral beam injection. The research program includes experimental evaluation of several possible plasma generation techniques, studies of plasma stability against magneto hydrodynamic (MHD) perturbations, testing of stabilization methods most suitable for a continuous discharge, studies on interaction between neutral gas and plasma and its influence on the overall energy confinement time. As a technology demonstration experiment, ALIANCE-T will feature several technical solutions envisioned for ALIANCE-1 [1] and succeeding machines such as modular magnet system with compact superconducting magnetic mirror solenoids, electrode-based plasma stabilization and limiting technologies suitable for a continuous discharge, continuous gas feed for plasma density control and cycling of neutral gas through pumping and gas feed systems. Successful completion of the experimental program is expected to provide well-defined solutions for the ALIANCE-1 experiment with neutral beam injection. The report presents general structure and parameters of the machine's subsystems, including the description of plasma source and its expected performance.

Key words: gas dynamic trap, fusion neutron source, superconducting magnets, plasma source.

DOI: $10.21517 / 0202-3822-2021-44-2-162-163$

ПРОЕКТ И ИССЛЕДОВАТЕЛЬСКАЯ ПРОГРАММА ЭКСПЕРИМЕНТА ALIANСЕ-Т

(краткое сообщение)

Д. Яковлев ${ }^{1}$, Т. Ахметов ${ }^{1}$, П. Багрянский ${ }^{1}$, Ц. Цзээн ${ }^{2}$, А. Иванов $^{1}$, И. Котельников ${ }^{l}$, Е. Кузьмин ${ }^{1}$, В. Приходько ${ }^{1}$, И. Шиховиев ${ }^{1}$ Ч. Чэнь ${ }^{2}$

\footnotetext{
${ }^{1}$ Институт ядерной физики им. Г.А. Будкера Сибирского отделения Российской академии наук, Новосибирск, 630090, Россия ${ }^{2}$ Институт технологии безопасности ядерной энергии, Хэфэйские институты физических наук, Китайская академия наук, Хэфэй, Аньхой, 230031, Китай
}

ALIANCE-T - пилотный эксперимент, направленный на проверку основных принципов работы объёмного термоядерного источника нейтронов ALIANCE [1] и изучение вопросов удержания плазмы в газодинамических пробочных ловушках, работающих в непрерывном режиме [2]. Исследовательская программа ALIANCE-T направлена на достижение непрерывного разряда в осесимметричном магнитном поле с высоким пробочным отношением ( 100) и параметрами плазмы, пригодными для инжекции нейтрального пучка. Программа исследований включает экспериментальную проверку нескольких возможных методов генерации плазмы, исследования устойчивости плазмы по отношению к магнитогидродинамическим (МГД) возмущениям, тестирование методов стабилизации, наиболее подходящих для непрерывного разряда, а также исследования взаимодействия нейтрального газа с плазмой и его влияния на общее время удержания энергии. В качестве эксперимента для демонстрации технологий ALIANCE-T представит несколько технических решений, которые предлагается применить в ALIANCE-1 [1] и последующих установках, таких как модульная магнитная система с компактными сверхпроводящими соленоидами магнитных пробок, технологии стабилизации и контроля границы плазмы при помощи электродов, пригодные 
для непрерывного разряда, непрерывная подача газа для поддержания плотности плазмы, а также замыкание газового цикла через системы откачки и подачи газа. Ожидается, что успешное выполнение экспериментальной программы позволит выработать готовые решения для эксперимента ALIANCE-1 с инжекцией нейтрального пучка. В отчёте представлены общая структура и параметры подсистем экспериментальной установки, включая описание источника плазмы и его ожидаемых характеристик.

Ключевые слова: газодинамическая ловушка, источник термоядерных нейтронов, сверхпроводящие магниты, источник плазмы.

\section{REFERENCES}

1. Bagryansky P.A. et al. - Nucl. Fusion, 2020, vol. 60, p. 036005.

2. Ivanov A.A., Prikhodko V.V. - Plasma Phys. Control. Fusion, 2013, vol. 55 p. 063001.

D.V.Yakovlev@inp.nsk.su 